\title{
Once Daily Sustained-Release Matrix Tablet of Naproxen: Formulation and In Vitro Evaluation
}

\section{Muhammad Rashedul Islam ${ }^{1}$, Ishtiaq Ahmed ${ }^{1}$, Mohiuddin Abdul Quadir ${ }^{2}$ and Md. Habibur Rahman ${ }^{1}$}

\author{
${ }^{1}$ Department of Pharmaceutical Technology, Faculty of Pharmacy, University of Dhaka, \\ Dhaka-1000, Bangladesh \\ ${ }^{2}$ Department of Pharmaceutical Chemistry, Faculty of Pharmacy, University of Dhaka, \\ Dhaka-1000, Bangladesh
}

\begin{abstract}
The objective of the present study was to develop once-daily sustained-release matrix tablets of naproxen, one of the most potent non-steroidal anti-inflammatory agents used in the treatment of arthritic pain. The tablets were prepared by direct compression method using hydrophilic matrix materials like Methocel $^{\circledR}$ K4M CR and Methoce $l^{\circledR}$ K15M CR. The tablets were subjected to measurement of thickness, diameter, weight variation, drug content, hardness and friability, the results of which were within compendial specification range. In vitro release studies were carried out by the USP basket method and were carried out at $\mathrm{pH} 7.4$ buffer for ten hours. The results of dissolution studies indicated that higher polymer content in the matrix (40\%) decreased the release rate of the drug as shown in formulation NMK4MF6 and NMK15MF6 (where lactose content is zero). The most successful formulations of the study, exhibited satisfactory drug release which was very close to the theoretical release profile. All the formulations exhibited diffusion-dominated drug release.
\end{abstract}

Key words: Naproxen, Methocel ${ }^{\circledR}$ K4M CR, Methocel $^{\circledR}$ K15M CR, Sustained release, Matrix tablets

\section{INTRODUCTION}

Rheumatoid arthritis and osteoarthritis are the most common arthritic diseases all over the world. Non-steroidal anti-inflammatory drugs are considered an important treatment options to reduce the pain and inflammatory properties. Naproxen, (S)-2-(6methoxynaphth-2-yl) propionic acid, a non-steroidal anti-inflammatory drug has been utilized from long time because of its efficacy and safety profile. Successful treatment means only when adequate blood levels are achieved and maintained with minimal variations. Sustained-release products have become important for the oral administration of many drugs because they give consistent optimal blood levels. Naproxen is extensively bound to plasma

Correspondence to:

Md. Habibur Rahman

E-mail: hrahman82@yahoo.com

Dhaka Univ. J. Pharm. Sci. 9(1): 47-52, 2010 (June) albumin, so it may be more efficient to deliver this drug in its sustained-release dosage form. ${ }^{1}$ The most commonly used method of modulating the drug release is to include it in a matrix system. ${ }^{2}$ Because of their flexibility, hydrophilic polymer matrix systems are widely used in oral controlled drug delivery to obtain a desirable drug release profile, cost effectiveness and broad regulatory acceptance. ${ }^{3}$ Hence, in the present work, an attempt has been made to develop once-daily sustained release matrix tablets of naproxen using hydrophilic matrix materials such as Methoce ${ }^{\circledR}$ K4M CR and Methocel ${ }^{\circledR}$ K15M CR. ${ }^{4}$

To obtain sustained release formulations, the presence of other excipients, such as fillers and lubricants, is usually required. In this study, the performance of several hydrophilic matrix systems

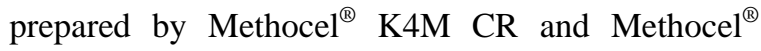
$\mathrm{K} 15 \mathrm{M}$ CR in controlling the release of naproxen was 
investigated and the effect of lactose in the formulations was observed on the release rate of naproxen.

\section{MATERIALS AND METHODS}

Naproxen was obtained as a gift sample from Roche Bangladesh Ltd. Methocel K4M ${ }^{\circledR}$ CR and

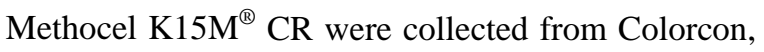
USA. Lactose (directly compressible grade, BDH, Germany), Magnesium stearate and Aerosil (colloidal Silicon dioxide) were supplied by Advance Chemical
Industries Limited; Bangladesh All reagents used in analysis were of analytical grade.

\section{Tablet Preparation:}

Different tablet formulations were prepared by direct compression technique (Table 1). All the powders were passed through ASTM (American Society of Testing and Materials) 80 mesh. Tablet formulations containing $500 \mathrm{mg}$ of naproxen, fillers, rate retarding polymers, binding agent and lubricants were prepared by double compressing the blended powders, using a Perkin-Elmer laboratory hydraulic press (London, UK) equipped with a $13 \mathrm{~mm}$ flat faced punch and die set at 5 ton pressure.

Table 1. Composition Of Naproxen Matrix Tablets.

\begin{tabular}{|c|c|c|c|c|c|c|c|c|c|c|c|c|}
\hline Formulation & I & II & III & IV & $\mathrm{V}$ & VI & VII & VIII & IX & $\mathrm{X}$ & XI & XII \\
\hline Naproxen & 500 & 500 & 500 & 500 & 500 & 500 & 500 & 500 & 500 & 500 & 500 & 500 \\
\hline METHOCEL ${ }^{\circledR}$ K4M CR & 20 & 50 & 100 & 150 & 180 & 200 & - & - & - & - & - & - \\
\hline METHOCEL ${ }^{\circledR}$ K15M CR & - & - & - & - & - & - & 20 & 50 & 100 & 150 & 180 & 200 \\
\hline Lactose & 180 & 150 & 100 & 50 & 20 & - & 180 & 150 & 100 & 50 & 20 & - \\
\hline Aerosil & 2 & 2 & 2 & 2 & 2 & 2 & 2 & 2 & 2 & 2 & 2 & 2 \\
\hline Mg-Stearate & 2 & 2 & 2 & 2 & 2 & 2 & 2 & 2 & 2 & 2 & 2 & 2 \\
\hline
\end{tabular}

\section{Evaluation of Tablets}

Thickness. The thickness of the tablets was determined using a thickness gauge (Mitutoyo, New Delhi, India). Six tablets from each batch were used and average values were calculated.

\section{Weight Variation Test}

To study weight variation, 20 tablets of each formulation were weighed using an electronic balance (OHAUS LS 200, Switzerland) and the average values were calculated.

\section{Hardness and Diameter}

For each formulation, hardness and diameter of 10 tablets were measured using the Electronic hardness tester (Erweka GMBH, DT6L Model, Germany).

In vitro Release Studies. The in vitro dissolution studies were carried out using USP apparatus type-I i.e. cylindrical basket in the metallic drive shaft (ERWEKA Model DT-700, Germany) rotated at a speed of $50 \mathrm{rpm}$ and the temperature was maintained at $37^{\circ} \pm 0.5^{\circ} \mathrm{C}$. The dissolution medium consisted of $0.1 \mathrm{~N}$ Hydrochloric acid for the first $2 \mathrm{~h}$ and the phosphate buffer $\mathrm{pH} 7.4$ from 3 to $10 \mathrm{~h}(900 \mathrm{ml})$, maintained at $37^{\circ} \pm 0.5^{\circ} \mathrm{C}$. The drug release at different time intervals was measured by UV Spectrophotometer (Shimadzu, Japan) at $331 \mathrm{~nm}$. It was made clear that none of the ingredients used in the matrix formulations interfered with the assay. The release studies were conducted in triplicate (6 tablets in each set) and the mean values were plotted versus time with SDs of less than 2, indicating the reproducibility of the results.

In vitro Drug Release Kinetics. In order to study the exact mechanism of drug release from the matrix tablets, drug release data was analyzed according to zero order, first order and Higuchi square root. ${ }^{5,6}$ The criterion for selecting the most appropiate model was chosen on the basis of goodness-fit-test. The dissolution data were also 
fitted according to the well-known exponential equation, which is often used to describe the drug release behavior from polymeric systems. ${ }^{7}$

\section{RESULTS AND DISCUSSION}

The naproxen matrix tablets of different formulations were evaluated for thickness, hardness, diameter and drug content. The thickness of the tablets ranged from $1.25 \pm 0.01$ to $1.26 \pm 0.02 \mathrm{~mm}$. The average percentage deviation of 20 tablets of each formula was less than $\pm 5 \%$. Drug content was found to be uniform among different batches of the tablets and ranged from $98.25 \pm 0.02$ to $99.69 \pm 0.04$. The hardness and diameter of the matrix tablets of all formulations ranged from $226.5 \pm 9.01$ to $289.5 \pm$ 5.42 and $12.94 \pm 0.01$ to $12.98 \pm 0.01 \mathrm{~N}$ respectively.

The results of dissolution studies of formulations F-I to F-VI composed of Methocel K4M and lactose are shown in figure 1. Tablets F-I, F-II, F-III, F-IV, F-V and F-VI released 4.54, 4.29, 5.13, 3.76, 2.82 and $2.25 \%$ of naproxen at the end of $2 \mathrm{hrs}$; and 98.27 , 82.56, 76.28, $66.14,62.02$ and $55.28 \%$ of drug at the end of 10 hrs. The formulations were further modified by replacing rate-retarding polymer Methocel ${ }^{\circledR}$ K4M CR (3000-5600 cps) with Methocel ${ }^{\circledR}$ K15M CR (11250-21000 cps).

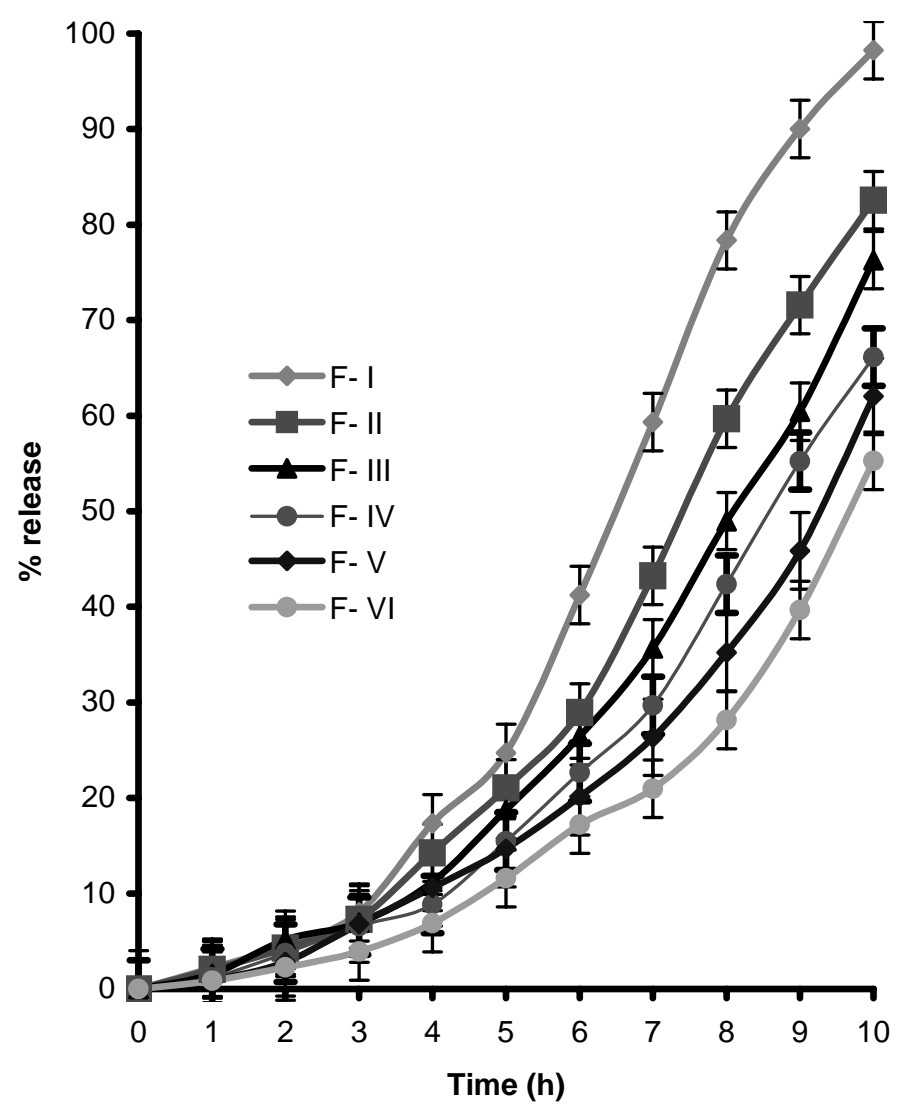

Fig. 1. Naproxen release profile from Methocel K4M CR matrix tablets. Lactose concentration used was F-I (36\%), F-II (30\%), F-III (20\%), F-IV (10\%), F-V (4\%) and F-VI (0\%) with respect to total tablet weight. Each point represents mean \pm SD; $n=6$.

The results of dissolution studies of formulations F-VII to F-XII composed of Methocel ${ }^{\circledR}$ K15M CR and lactose are shown in figure 2. The tablet formulations of F-VII, F-VIII, F-IX, F-X, F-XI and F-XII released 4.59\%, 3.76\%, 2.44\%, 4.50\%, 2.63\% and $2.87 \%$ of naproxen at the end of $2 \mathrm{hrs}$; and 89.59 , $78.48,66.87,58.89,49.12$ and $40.54 \%$ of drug at the end of 10 hrs. Incorporation of lactose along with drug and polymer modify the release of naproxen from the matrix tablet formulations. ${ }^{8}$ For all the 
formulations, the release rate of naproxen was extremely low at low acidic $\mathrm{pH}$, since after 2 hours only less than $6 \%$ of drug was released from different matrix tablets. The rate and extent of drug release was found to be inversely related to the level of Methocel $^{\circledR}$ K4M CR and Methocel ${ }^{\circledR}$ K15M CRpresent in the matrix structure, i.e., the drug release increased with decrease in the polymeric content of the matrix. Such increase in the polymer content results in a decrease in the drug release rate due to a decrease in the total porosity of the matrices (initial porosity plus porosity due to dissolution of the drug). Preferential solubility of lactose in the dissolution media decreased the diffusion pathlength of the drug from the tablet, which was synergistically affected by swelling pressure of the hydrophilic polymer upon the matrix structure., 9

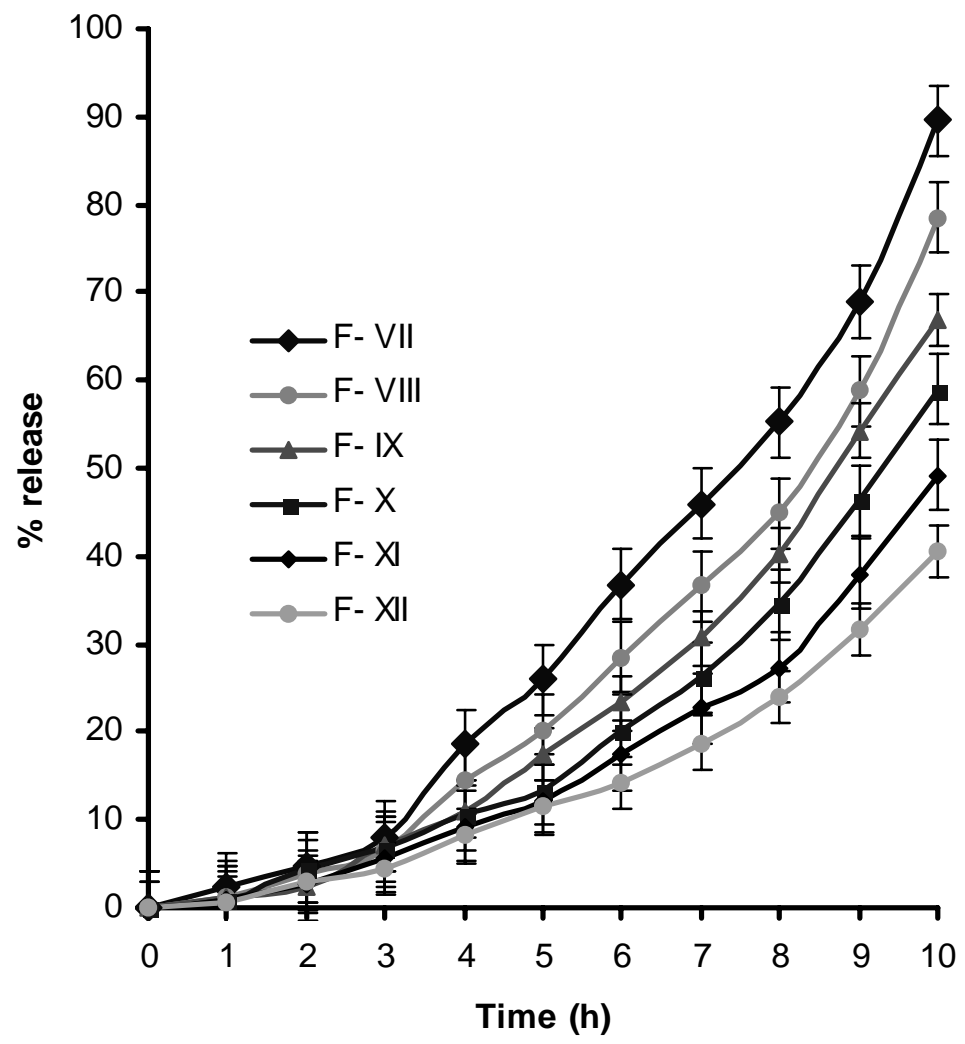

Fig. 2. Naproxen release profile from Methocel K15M CR matrix tablets. Lactose concentration used was F-I (36\%), F-II (30\%), F-III (20\%), F-IV (10\%), F-V (4\%) and F-VI (0\%) with respect to total tablet weight. Each point represents mean \pm SD; n=6.

Different kinetic equations (zero-order, firstorder and Higuchi equation) were applied to interpret the release rate of naproxen from the matrix. The best fit with higher correlation $\left(r^{2}>0.98\right)$ was found with zero order equation for all the formulations. The dissolution data were also fitted according to the well-known exponential equation, which is often used to describe the drug release behavior from polymeric systems. The values of release exponent (n), kinetic rate constant $\left(\mathrm{K}_{1}\right.$ and $\left.\mathrm{K}_{2}\right)$ and correlation
- coefficient values $\left(\mathrm{r}^{2}\right)$ as calculated from the equation and are presented in the Table 2.

For a tablet having cylindrical shape, different values of ' $\mathrm{n}$ ' indicate different release pattern ; $\mathrm{n}=$ 0.45 for Fickian (Case I) release, $>0.45$ but $<0.89$ for non - Fickian (anomalous) release and 0.89 for Case II (Zero order) release and $>0.89$ for Case II From super case II type of release. From the table, we can see that most of the values of $\mathrm{n}$ are close to 0.7 , which is a clear indication of anomalous or nonfickian transport. ${ }^{10}$ This is always true for HPMC 
based systems. Only F-III formulation demonstrated drug release mechanism by Fickian (case I) transport as observed from the $\mathrm{n}$ values, which is close to 0.45 . This is an unusual observation based on the fact that F-III contains substantially larger amount of hydrophilic polymers. Nonetheless, as non-Fickian type of release is a contribution from both diffusion controlled and swelling controlled mechanisms, it is not completely unlikely that at a particular ratio of HPMC to soluble filler generates matrix type structure which facilitates drug release more by diffusion mediated mechanism. It should be noted that, for diffusion to occur the first step is wetting of drug by water followed by its dissolution so that the drug molecule is available in molecular form to diffuse out of the matrix. ${ }^{11}$ Hence the net release observed is a cumulative effect of drug solubility (influenced by its structure, molecular weight and pKa), polymer property (hydrophilicity/lipophilicity, molecular weight and tortuosity) and the relative ratio of drug and polymer in the dosage form. ${ }^{12}$

Table 2. Correlation - coefficient $\left(r^{2}\right)$, release exponent $(n)$, kinetic rate constants $\left(k_{1}\right.$ and $\left.k_{2}\right)$ values for naproxen matrix tablets

\begin{tabular}{ccccc}
\hline $\begin{array}{c}\text { Formulation } \\
\text { code }\end{array}$ & $\mathrm{n}$ & $\mathrm{r}^{2}$ & $\boldsymbol{K}_{1}$ & $\boldsymbol{K}_{2}$ \\
\hline I & 0.657 & 0.897 & 1.02 & 2.88 \\
II & 0.765 & 0.994 & 1.09 & 2.67 \\
III & 0.432 & 0.981 & 1.76 & 2.62 \\
IV & 0.732 & 0.996 & 1.76 & 2.43 \\
V & 0.675 & 0.978 & 1.85 & 2.42 \\
VI & 0.761 & 0.993 & 1.99 & 2.11 \\
VII & 0.776 & 0.997 & 1.09 & 2.09 \\
VIII & 0.765 & 0.995 & 1.87 & 2.89 \\
IX & 0.789 & 0.987 & 1.76 & 2.77 \\
X & 0.712 & 0.983 & 1.90 & 2.65 \\
XI & 0.743 & 0.983 & 1.95 & 2.54 \\
XII & 0.789 & 0.991 & 1.98 & 2.43 \\
\hline
\end{tabular}

Values of $\mathrm{n}$ for all matrices studied here demonstrate drug release through anomalous behavior due to the presence of increasing amount hydrophilic polymers. This is to mention that, anomalous behavior corresponds to sum contribution from diffusion, erosion and swelling-controlled mechanisms. In all these matrices availability of the water within the gel structure is also limited, and therefore a dissolution-controlled release is also involved. As the viscosity of the HPMC polymer increased in the Methocel grade, the release rate of Naproxen showed a tendency to decrease. It has been postulated that, increasing the molecular weight or viscosity of the polymer in a matrix formulations increases the gel layer viscosity and thus slows down drug dissolution. ${ }^{13}$ Also the greater the viscosity of the gel, the more resistant the gel to dilution and erosion, thus controlling the drug dissolution.
Generally the penetration rate of water in matrices containing hydrophilic polymer is determined by the equilibrium between promotive forces of admission of water and those that act against its admission, i.e. the viscosity forces. In so far as the movement of drugs like Naproxen through gelatinous layer is controlled by the diffusion, the process gets slower in more viscous medium that is developed around the dosage form.

From the kinetic rate constants of $\mathrm{K}_{1}$ and $\mathrm{K}_{2}$ values where $K_{1}$ is the constant indicating the extent of swelling controlled mechanism and $\mathrm{K}_{2}$ gives the extent of diffusion or Fickian mechanism controlled transport process. All the formulations containing Methocel $^{\circledR}$ K4M CR and Methocel ${ }^{\circledR}$ K15M Cr showed that, the $K_{1}$ value is less than $K_{2}$, it is decisive that the release is predominantly controlled by diffusion-controlled process. Thus preparation of 
Naproxen matrix tablets with Methocel polymers as rate controlling agent were found to be promising for controlling the release of Naproxen. Incorporation of lactose in various ratios with the polymers enhanced the release rate controlling process. The prepared tablets exhibited zero order kinetics and the permeation profile was matrix diffusion type. In conclusion, results of the present study suggest sustaining matrix-forming ability of Methocel with variations with lactose could be used for sustained release drug delivery of naproxen.

\section{ACKNOWLEDGEMENTS}

The authors are thankful to the Advanced Chemical Industries Ltd., Bangladesh for providing the necessary support to complete the work.

\section{REFERENCES}

1. Iqbal, Z. and Babar, A. 2002. Controlled-release naproxen using micronized ethyl cellulose by wet-granulation and solid-dispersion method. Drug Dev. Ind. Pharm. 28, 129-34.

2. Zaghloul, A. A., Khan, M. A. and Vaithiyalingam, S. R. 2001. Response surface methodology to obtain naproxen controlled release tablets from its microspheres with Eudragit L100-55. J Microencapsul. 18, 651-62.

3. Amaral,M. H. and Ferreira, D. C. 2001. Naproxen availability from variable-dose and weight sustained-release tablets. Drug Dev. Ind. Pharm. 27, 221-25.

4. Harland, R. S.., Gazzaniga, A., Sangalli, M. E., Colombo, P., and Peppas N. A. 1988Drug/polymer matrix swelling and dissolution. Pharm. Res. 5, 488-494.
5. Higuchi. T. 1963. Mechanism of sustained action medication, theoretical analysis of rate of release of solid drugs dispersed in solid matrices. J. Pharm. Sci. 52, 1145-1149.

6. Roseman, T. J. and Highuchi, W. I. 1970. Release of medroxyprogesterone acetate from a silicone polymer. J.Pharm. Sci. 59, 353.

7. Beren, A. R., and Hopfenberg, H. B.1978. Diffusion relaxation in glassy polymer powders: Separation of diffusion and relaxation parameters. Polymer 19, 489-496.

8. Reza, M. S., Quadir, M. A. and Haider, S. S. 2005. Comparative evaluation of plastic, hydrophobic and hydrophilic polymers as matrices for controlled release drug delivery. J. Pharm. Pharmaceut. Sci. 6, 282-291.

9. Ju, R.T.C., Nixon, P.R.and Patel, M.V. 1995. Drug release from hydrophilic matrices 1 . New scaling laws for predicting polymer and drug release based on the polymer disentanglement concentration and the diffusion layer. $J$. Pharm. Sci. 84, 1455-1463.

10. Korsmeyer, R. W., Gurny, R., Doelker, E., Buri, P. and Peppas, N. A. 1983. Mechanisms of solute release from porous hydrophilic polymers. Int. J. Pharm. 15, 25.

11. Devi, P., Ranga Rao, K. V. and Buri P.1990. Influence of molecular size and water solubility of the solute on its release from swelling and erosion controlled polymeric matrixes. $J$. Controlled Release, 12, 133-141.

12. Langer, R.S. and Peppas, N.A. 1981. Present and Future applications for biomaterials in controlled drug delivery systems. Biomaterials. 2, 201-214.

13. Jain,R, Shah, N.H., Malick, A.W. and Rhodes, C.T. 1998. Controlled drug delivery by biodegradable poly (ester) devices: different preparative approaches. Drug Develop. Ind. Pharm. 24, 703-27. 